\title{
Hybrid sol-gel-conducting polymer synthesised by electrochemical insertion: tailoring the capacitance of polyaniline
}

\author{
F. Montilla, ${ }^{*}$ M. A. Cotarelo and E. Morallón \\ Received 1st August 2008, Accepted 13th October 2008 \\ First published as an Advance Article on the web 20th November 2008 \\ DOI: 10.1039/b813323a
}

The templated growth of polyaniline through porous sol-gel films on ITO substrates has been performed. The polyaniline-silica composites have been synthesized by electrochemical reactive insertion in potentiodynamic and potentiostatic conditions. When the polymer is synthesized by potential step experiments, the shape of the chronoamperograms indicates two regimes for the electrochemical polymerization of PANI. Electropolymerization happens faster within the silica pores than on bare ITO electrodes due to confinement effects of oligomeric species formed upon oxidation. The electrochemical capacitance of the silica-PANI hybrids is several time higher than the capacitance of ITO/PANI synthesized under the same conditions. The silica matrix avoids the electric collapse between vicinal conducting fibres in ITO/silica-PANI but allows the diffusion of ionic species that are in contact with the conjugated polymer, increasing therefore the conducting surface exposed to the electrolytic solution.

\section{Introduction}

Hybrid nanomaterials made of conjugated polymers inside porous silica combine the special properties of an inorganic matrix (chemical stability, tailorable morphology and porosity, optical transparency, physical strength) with the electrical or optical properties of organic conjugated polymers and have a wide range of applications, such as sensory devices, microelectronics or photonics. ${ }^{1-7}$

Silica matrices are usually synthesised by means of sol-gel techniques. The porosity and morphology of these types of materials are easily modulated by the processing conditions and the composition of the precursor solution $(\mathrm{pH}$, aging of the sol, drying, presence of surfactants, etc.). ${ }^{8}$ The morphology and porosity of the silica have a great influence on its properties, influencing its applications as solid electrolytes or sensory devices, among others. ${ }^{9-12}$

The synthesis of hybrid silica-conducting polymers can be performed by several methods, i.e., incorporation of the polymer after the synthesis of the inorganic matrix, using thermal, pressure, or electrophoretical deposition (heterogeneous insertion), introduction of the polymer in the precursor solution of the silica gel (homogeneous insertion), polymerisation of the monomer included in the porous matrix, performing the reaction by chemical or electrochemical methods (reactive insertion).

One of the most studied conjugated polymers is polyaniline because of its especial properties: thermal and electrochemical stability, easy and cheap fabrication, high conductivity in doped form, fast and stable transition between doped and reduced states, electrochromism, etc. One of the most important electrochemical applications of this polymer is charge storage. ${ }^{13,14}$ This polymer can be considered as a potential

Departamento de Química Física e Instituto Universitario de Materiales de Alicante, Universidad de Alicante, Apdo. de Correos 99, E03080 Alicante, Spain. E-mail: francisco.montilla@ua.es candidate for electrochemical capacitor materials because it presents a continuous range of oxidation states with increasing electrode potential.

A silica/conducting polymer composite could improve the performance of supercapacitors, taking advantage of both the double layer pseudo-capacitative contribution from the conducting polymer and an improvement in the mechanical properties and stability from the inorganic matrix. ${ }^{15}$

Due to the low processability of PANI, reactive insertion is the most promising method for the incorporation of this polymer inside the inorganic matrix. ${ }^{9,16,17}$ The electrochemical synthesis has additional advantages for the synthesis of interpenetrating polymer-silica composites. The polymers grow from the conducting support through the pores of the overlying silica structure. Easy control of the amount of PANI deposited, of the oxidation state of the polymer and of morphology can be attained with electropolymerization methods. ${ }^{18,19}$ Although we have focused our attention on polyaniline, this method of synthesis can be applied for the preparation of other conducting polymer-silica hybrids, which could be synthesized by electropolymerization, such as polypyrrole or polythiophene, among others.

This work studies the electrochemical reactive insertion of polyaniline though microporous silica. The effect of the silica matrix on the electrochemical reactivity and the properties of the resultant interpenetrating polymeric composite material have been explored.

\section{Experimental part}

The chemicals used in this work were all reagent grade. Aniline was supplied by Merck and was distilled before use. Tetraethyl orthosilicate (TEOS) was purchased from Sigma-Aldrich.

The electrolytic medium employed in the voltammetric experiments was $0.5 \mathrm{M} \mathrm{H}_{2} \mathrm{SO}_{4}$ prepared with $18.2 \mathrm{M} \Omega \mathrm{cm}$ 
resistivity water. The electrochemical cells were purged by bubbling a $\mathrm{N}_{2}$ flow for $20 \mathrm{~min}$, and the $\mathrm{N}_{2}$ atmosphere was maintained during all the experiments. The counter electrode was a platinum wire. All potentials were measured against a reversible hydrogen electrode (RHE) immersed in the test solutions and are presented in this scale.

Indium-tin oxide (ITO) covered glass substrates $\left(60 \mathrm{~S} \mathrm{~cm}^{-1}\right)$ were used as working electrodes for the electrochemical synthesis-deposition of the conducting polymer. Prior to its use, the ITO glass was degreased by sonication in an acetone bath and rinsed with an abundance of deionised water.

The silica precursor solution was prepared by mixing $5.6 \mathrm{ml}$ of TEOS with $7.6 \mathrm{ml}$ of ethanol and $2.7 \mathrm{ml}$ of $0.01 \mathrm{M} \mathrm{HCl}$. The mixture was stirred in a closed vial for three hours. The sol-gel films on ITO substrates were prepared by a dip-casting method. The electroactive areas exposed to the solution were determined by means of cyclic voltammetry in a solution of $0.5 \mathrm{M} \mathrm{H}_{2} \mathrm{SO}_{4}$, assuming a value of specific capacitance for the doped tin oxide of $8 \mu \mathrm{F} \mathrm{cm}$ c. $^{-20}$

The electrochemical equipment was an Autolab PGSTAT30 equipped with a SCAN-GEN module. The low-frequency capacitance measurements were performed by potential cycling at different scan rates ranging from 5 to $100 \mathrm{mV} \mathrm{s}^{-1}$. The slope of the variation of current density at the doping peak of PANI as a function of the scan rate corresponds to the capacitance for that system. This method is similar to that employed in the determination of the capacitance of porous tin oxide electrodes presented in a previous paper. ${ }^{20}$

The surface texture of the electrodes was studied by scanning electron microscopy (SEM Hitachi S-3000N). A series of samples was submitted for cryo-SEM analysis in order to determine the morphology of the hydrogels synthesised. An ITO/silica electrode placed onto a holder of a cryotransfer system was plunged into subcooled liquid nitrogen, and then transferred to a preparation unit via an air lock transfer device. The frozen specimens were transferred directly via a second air lock to the microscope cold stage and etched for $5 \mathrm{~min}$ at $-90{ }^{\circ} \mathrm{C}$. After ice sublimation and in the preparation unit, the etched surfaces were sputter coated with gold for $4 \mathrm{~min}$ at $10 \mathrm{mV}$. Samples were transferred to the scanning electron microscope chamber microscope at $-150{ }^{\circ} \mathrm{C}$.

\section{Results}

In order to determine the actual morphology of the silica hydrogels, ITO/silica samples were submitted to a cryo-SEM analysis. Fig. 1 shows the silica gel deposited onto an ITO electrode after freezing in liquid nitrogen. The silica layer shown in
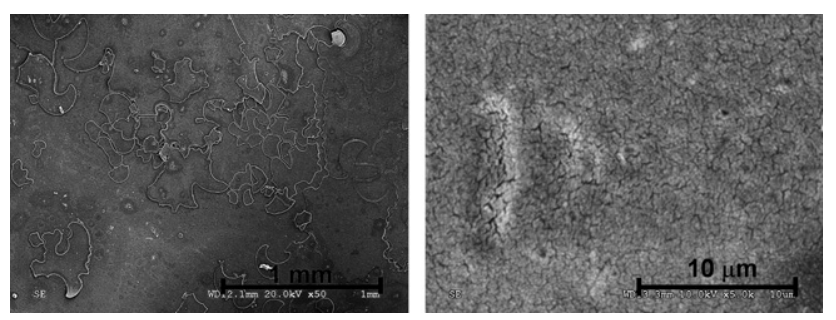

Fig. 1 Cryo-SEM image of an ITO/silica electrode.
Fig. 1a presents a heterogeneous aspect with the presence of irregular and cracked plates. Fig. 1b shows in more detail the electrode surface, which presents a cracked surface with submicrometric pores (lower than $200 \mathrm{~nm}$ in size).

\subsection{Electrochemical synthesis of silica-PANI composites}

An ITO electrode covered with porous silica was immersed in an electrochemical cell containing $0.5 \mathrm{M} \mathrm{H}_{2} \mathrm{SO}_{4}+0.1 \mathrm{M}$ aniline at a controlled potential of $0.3 \mathrm{~V}$. The electrode remained immersed at this potential for 2 minutes to facilitate the diffusion of the aniline monomers inside the silica pores to reach the underlying ITO surface. Fig. 2a shows the voltammogram obtained at potentials between +0.3 and $+1.1 \mathrm{~V}$.

An oxidation current is observed in the initial scan at potentials more positive than $+1.05 \mathrm{~V}$, related to the oxidation of aniline monomer. In the successive scans, the growth of anodic and cathodic currents in that zone of potentials is indicative of the growth of electroactive species on the electrode. After 10 cycles, the resultant ITO/silica-PANI electrode was emersed from the cell at a controlled potential of $+0.6 \mathrm{~V}$. The electrode was rinsed with deionised water in order to remove the adsorbed unreacted aniline species. This electrode was transferred to an electrochemical cell containing $0.5 \mathrm{M} \mathrm{H}_{2} \mathrm{SO}_{4}$ in the absence of monomer to test its electrochemical response. Fig. $2 \mathrm{~b}$ shows the steady cyclic voltammogram of the ITO/silica-PANI composite electrode. The first oxidation peak appears at $+0.47 \mathrm{~V}$ during the positive-going potential scan, related to the well-known transition of the polyaniline from its semiconducting-state (leucoemeraldine) to the conductive form (emeraldine). Additional oxidation peaks appear around $+0.7 \mathrm{~V}$ corresponding to the redox processes associated with the overoxidation of the polymer followed by hydrolysis to quinone-type species. ${ }^{21}$ The current arising from $+0.9 \mathrm{~V}$ is due to the second redox transition of polyaniline from the emeraldine to the pernigraniline form. During the reverse scan, the corresponding counter-processes can be observed.

The electrochemical synthesis of polyaniline was also performed by potentiostatic step experiments. Fig. 3 shows a typical chronoamperometric experiment for an ITO electrode (solid line)
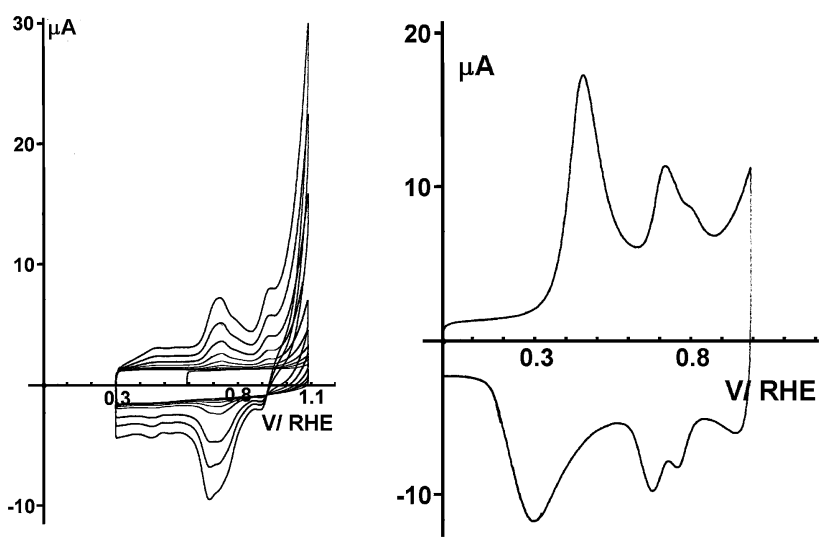

Fig. 2 (a) Successive cyclic voltammograms of an ITO/silica electrode in a solution of $0.5 \mathrm{M} \mathrm{H}_{2} \mathrm{SO}_{4}$ and $0.1 \mathrm{M}$ aniline. (b) Steady-state cyclic voltammograms of the resultant ITO/silica-PANI electrode in a solution of $0.5 \mathrm{M} \mathrm{H}_{2} \mathrm{SO}_{4}$. Scan rate $100 \mathrm{mV} \mathrm{s}^{-1}$. 


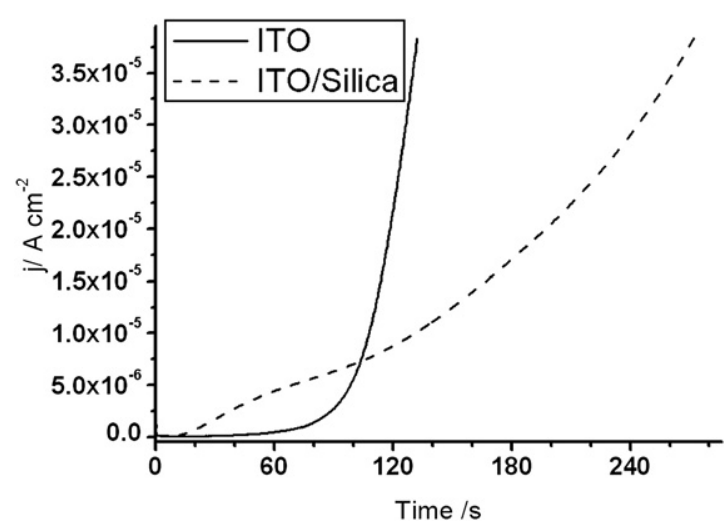

Fig. 3 Potentiostatic step experiments of ITO (solid line) and ITO/silica (dashed line) electrodes in a solution of $0.5 \mathrm{M} \mathrm{H}_{2} \mathrm{SO}_{4}$ and $0.1 \mathrm{M}$ aniline. Initial potential: $+0.6 \mathrm{~V}$, final potential: $+1.05 \mathrm{~V}$.

and an ITO/silica electrode (dashed line). An exponential growth of the current can be observed for the conventional ITO electrode. The shape of this plot is related to the autocatalytic effect of polyaniline (pernigraniline) for the oxidation of the new monomer units ${ }^{22}$ and the increase of the electroactive surface area. The high rate of current from (exponential factor of $0.058 \mathrm{~s}^{-1}$ ) indicates the formation of a great density of growing PANI nuclei in the electrode surface.

Quite different behaviour can be observed during the experiment performed with the ITO/silica electrodes. Two different current regimes can be observed in the chronoamperometric curve separated by an inflection point at $77 \mathrm{~s}$. The concave shape of the initial part of the curve does not fit an exponential growth but, in the initial stages of electropolymerization, the current density is higher than in the case of the conventional ITO electrode. From $77 \mathrm{~s}$, the convex shape of the curve can be fitted to an exponential growth although with a growing rate $\sim 4$ times lower than on bare ITO electrodes (exponential factor $0.014 \mathrm{~s}^{-1}$ ). The reasons for the different growth regimes of PANI deposits will be discussed later.

For comparative purposes, similar conditions of synthesis were applied for the growth of polyaniline on bare ITO and on ITO/silica electrodes. Potentiostatic step experiments to $1.05 \mathrm{~V}$ in $0.1 \mathrm{M}$ aniline were adjusted to obtain a similar amount of total charge density passed though both types of electrodes in order to obtain comparable amounts of polyaniline deposited. The PANI modified electrodes were tested in a solution in the absence of monomer. Fig. 4 shows the steady voltammograms of ITO/ PANI and ITO/silica-PANI electrodes with $0.34 \mu \mathrm{g} \mathrm{cm}^{-2}$ PANI electrodeposited.

The voltammetric charge of the composite silica-PANI is higher than the equivalent electrode without silica. This is due to differences in the surface area of PANI exposed to the electrolytic solution. PANI grown through the silica pores presents a higher electroactive surface than the equivalent electrode in the absence of the inorganic matrix.

\subsection{Capacitance measurements}

The capacitance of the electrodes was determined by potential cycling at different scan rates ranging from 5 to $100 \mathrm{mV} \mathrm{s}^{-1}$. A

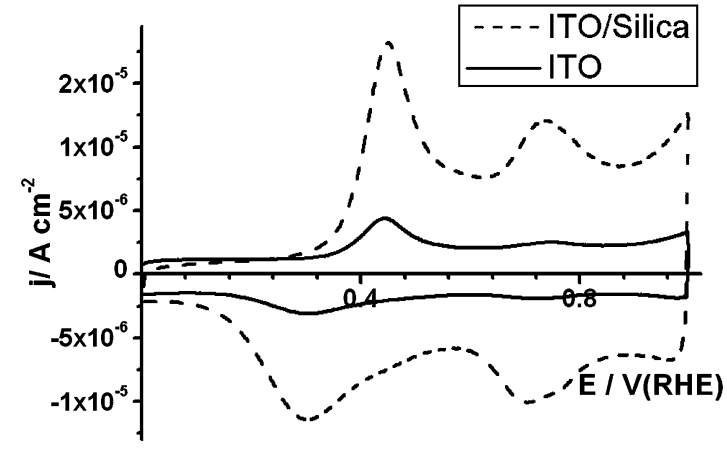

Fig. 4 Steady-state cyclic voltammograms of the resultant ITO/PANI (solid line) and ITO/silica-PANI (dashed line) electrodes in a solution of $0.5 \mathrm{M} \mathrm{H}_{2} \mathrm{SO}_{4}$. The amount of PANI deposited was $0.34 \mu \mathrm{g} \mathrm{cm}^{-2}$ for both electrodes. Scan rate $100 \mathrm{mV} \mathrm{s}^{-1}$.

representation of the current density at the doping peak around $+0.45 \mathrm{~V}$ vs. the scan rate is shown in Fig. 5 for both electrodes. The linear plot obtained confirms the capacitative character of the current related to the doping process of polyaniline. The scan rate-averaged capacitance for each electrode is obtained from the slope of the linear plots. As can be observed, the slope is higher for the hybrid silica-PANI electrode. The capacitance values for several electrodes are presented in Table 1.

The values of specific capacitance and mass capacitance of the silica-PANI composites are higher than the equivalent ITO/ PANI electrodes. This is due to a larger surface exposed to the solution in the interpenetrating polymer-silica composites. The maximum mass capacitance obtained is $606.9 \mathrm{~F} \mathrm{~g} \mathrm{~g}^{-1}$, corresponding to the sample S2 with an amount of $0.588 \mu \mathrm{g}$ of PANI deposited per unit surface area, which is a mass capacitance $\sim 7$ times higher than the equivalent ITO/PANI electrode.

Fig. 6 plots the capacitance of ITO/PANI and ITO/silicaPANI electrodes against the amount of deposited polymer. In both type of samples, the specific capacitance (Fig. 6a) increases with the amount of polymer deposited, although the values in the

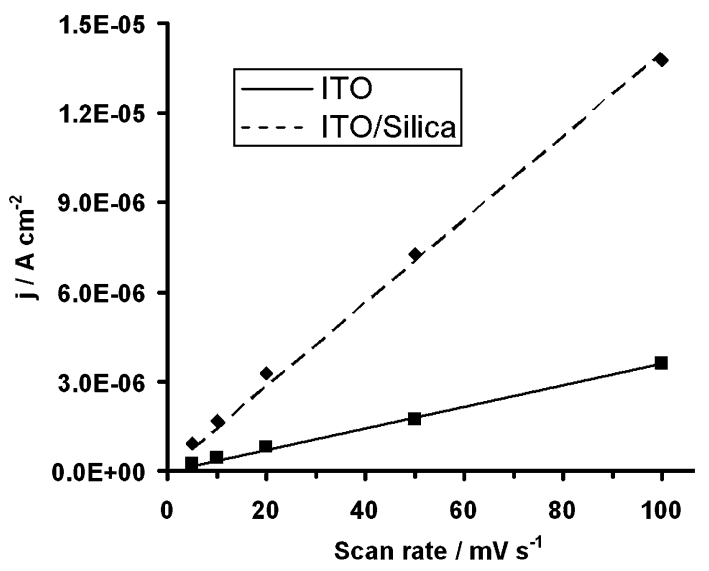

Fig. 5 Evolution of the current density at $+0.45 \mathrm{~V}$ against the potential scan rate for ITO/PANI (solid line) and ITO/silica-PANI (dashed line) electrodes in a $0.5 \mathrm{M} \mathrm{H}_{2} \mathrm{SO}_{4}$ solution. The potential scan rate ranges from 5 to $100 \mathrm{mV} \mathrm{s}^{-1}$. 
Table 1 Amount of polyaniline deposited, specific and mass capacitance and defects of several ITO/PANI (label I) and ITO/silica-PANI (label S) electrodes. Data obtained from experiments performed as in Fig. 5

\begin{tabular}{llccc}
\hline Label & PANI deposited ${ }^{a} / \mu \mathrm{g} \mathrm{cm}^{-2}$ & Specific capacitance ${ }^{b} / \mu \mathrm{F} \mathrm{cm}^{-2}$ & ${\text { Mass capacitance/F } \mathrm{g}^{-1}}^{\text {Defects }^{c}}$ \\
\hline I1 & 0.342 & 27.8 & 81.3 & 0.14 \\
I2 & 0.949 & 85.2 & 89.7 & 0.04 \\
I3 & 1.363 & 301.2 & 220.9 & 0.06 \\
I4 & 2.324 & 605.8 & 260.7 & 0.06 \\
S1 & 0.347 & 130.1 & 380.5 & 0.30 \\
S2 & 0.588 & 357.0 & 596.3 & 0.39 \\
S3 & 0.984 & 586.9 & 464.4 & 0.37 \\
S4 & 1.367 & 634.6 & 312.0 & 0.24
\end{tabular}

${ }^{a}$ Assuming a value of 1 electron per deposited aniline monomer. ${ }^{b}$ Calculated with the real surface area of ITO. ${ }^{c}$ Ratio of the oxidation peak current at $+0.7 \mathrm{~V}$ and at $+0.45 \mathrm{~V}$.

a

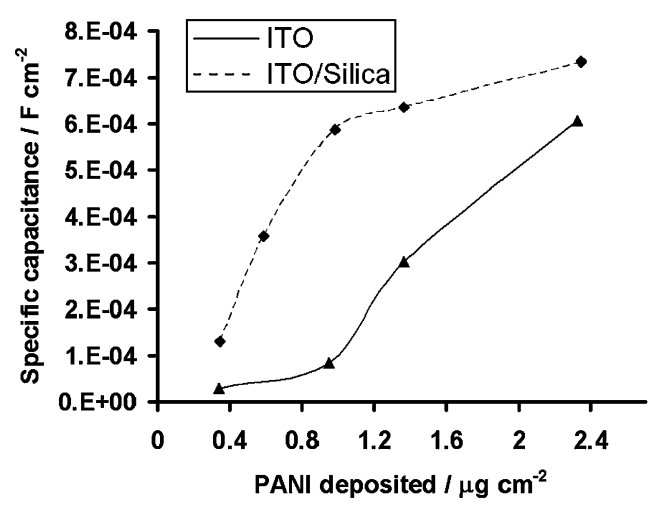

b

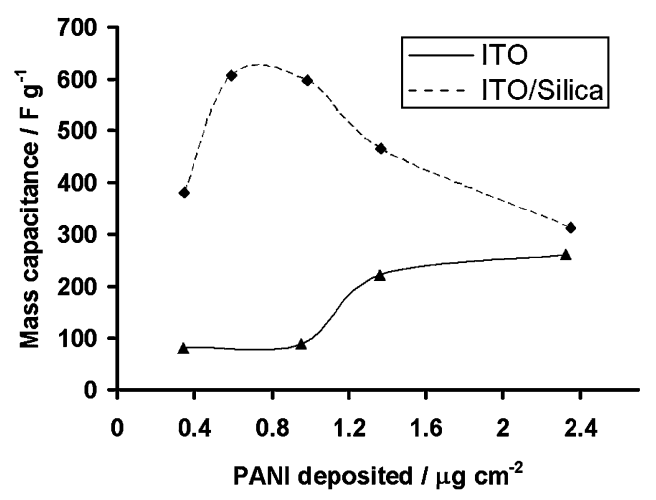

Fig. 6 Evolution of the specific capacitance (a) and mass capacitance (b) as a function of the amount of deposited PANI.

silica-PANI composites are always higher than the corresponding samples deposited onto bare ITO electrodes.

The trends of the curves are related to the different areas of PANI exposed to the electrolytic solution. Fig. $6 \mathrm{~b}$ shows the evolution of the mass capacitance with the amount of deposited polymer, which is an indication of the area of PANI in contact with the solution. For values of PANI deposited of less than $1 \mu \mathrm{g}$ $\mathrm{cm}^{-2}$, the value of capacitance is at a maximum for the silicaPANI hybrids, while the capacitance remains low in the ITO/ PANI samples. For values of PANI more than $1 \mu \mathrm{g} \mathrm{cm}^{-2}$, the mass capacitance of the hybrid decreases and the values converge for both types of electrodes.

The presence of silica matrices on the ITO electrodes induces not only changes in the electroactive area of deposited PANI, but also in the chemical structure of the electrodeposited polymer. The presence of redox processes around $+0.7 \mathrm{~V}$ in the voltammograms of the PANI modified electrodes (see Fig. 2b and $4 \mathrm{~b}$ ) indicates the presence of quinone-type defects in the polymers.

The amount of defective sites was estimated as a quotient between the oxidation current at $+0.7 \mathrm{~V}$ (defects peak) divided by the current at $+0.45 \mathrm{~V}$ (doping peak) and is indicated in Table 1 . The electrosynthesized PANI in the silica matrix presents a higher amount of defective sites. The amount of defects is higher in the composite samples, especially those with amounts of PANI deposited, lower than $1 \mu \mathrm{g} \mathrm{cm}^{-2}$. The reasons for this effect will be discussed later.

\subsection{Morphological characterization}

In order to rationalize the results obtained, the morphology of the PANI modified electrodes was checked by SEM microscopy. Fig. 7 shows the SEM micrographs of an ITO/PANI electrode synthesised with a potentiostatic step at $+1.05 \mathrm{~V}$. Several islands of polyaniline are regularly distributed at distances around 20$60 \mu \mathrm{m}$ for the electrode labelled as I2 in Table 1. Fig. 7a shows in detail some of those islands. Their diameter ranges from 3 to $7 \mu \mathrm{m}$, although smaller polyaniline deposits around the agglomerated zones with diameters near $1 \mu \mathrm{m}$ can be observed. The agglomerates are composed of several submicrometric PANI particles, as can be observed in detail in Fig. 7b, with diameters of $300 \mathrm{~nm}$ or lower.

Fig. 7c shows the aspect of a PANI layer grown extensively in a potentiostatic step for 20 minutes at $+1.05 \mathrm{~V}$. The image shows a regular fibrilar aspect of the PANI deposit. The fibres are quite uniform with a diameter around $300 \mathrm{~nm}$.

Fig. 8 shows the SEM images of several ITO/silica-PANI composites. 
a

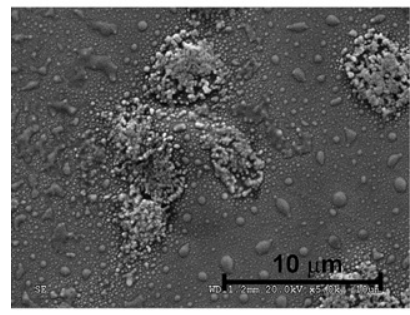

c

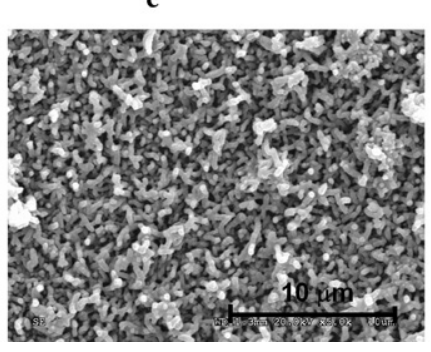

Fig. 7 SEM images of ITO/PANI electrodes. (a) and (b) I2 sample from Table 1. (c) ITO/PANI electrode obtained in a potentiostatic step of $20 \mathrm{~min}$ at $+1.05 \mathrm{~V}$.

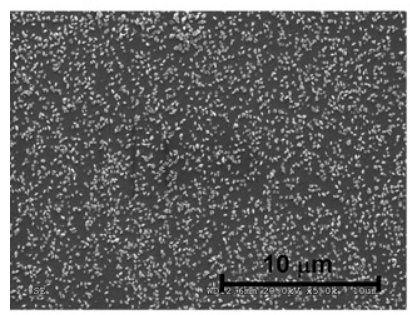

c

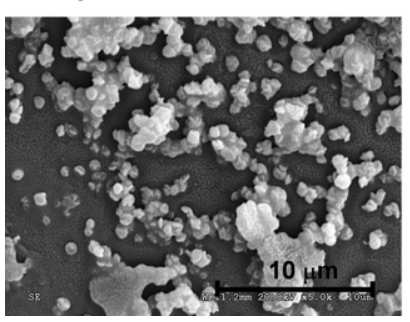

b

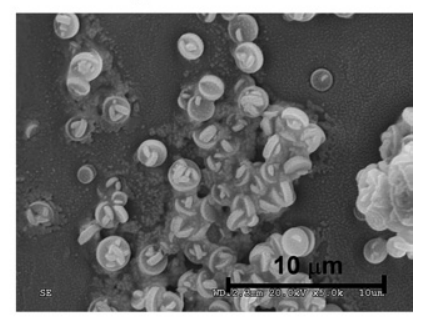

d

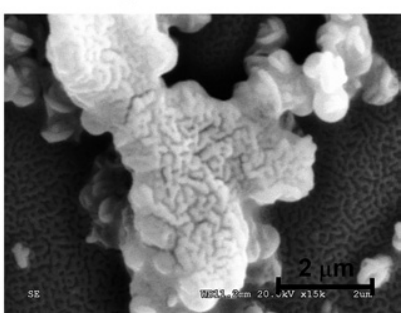

Fig. 8 SEM images of several ITO/silica-PANI electrodes labelled S3 (a), S4 (b) and S5 (c, d) in Table 1.

Fig. 8a corresponds to the composite with $0.984 \mu \mathrm{g} \mathrm{cm}^{-2}$ of PANI (sample S3). This sample has the same amount of PANI deposited as the electrode shown in Fig. 7a. Several nuclei can be found on the silica deposit. The emergent round-shaped PANI have sizes ranging from $8 \mathrm{~nm}$ to $200 \mathrm{~nm}$, however, there is no agglomeration of the deposited PANI in contrast to the equivalent ITO/PANI electrode in the absence of the inorganic matrix (Fig. 7a).

Fig. $8 \mathrm{~b}$ shows the SEM image of another composite electrode (S4) with a higher amount of PANI. Two types of PANI particles can be found. Circular-shaped PANI deposits with diameters ranging 1 to $2 \mu \mathrm{m}$ and incipient fibrils are starting to grow over those particles. Around the large PANI deposits, some particles with smaller sizes appear with similar sizes to that observed in the previous electrode (Fig. 8a).

Fig. 8c shows the ITO/silica-PANI (S5) electrode with $2.35 \mu \mathrm{g}$ $\mathrm{cm}^{-2}$. Although there are still similar circular-shaped particles, as in electrode S4, several zones present PANI agglomerates. One of those agglomerates is shown in detail in Fig. 8d. In that image, there is evidence of the formation of PANI fibrils of diameters around $200-300 \mathrm{~nm}$, similar in size to the fibrils obtained in a conventional ITO/PANI electrode (Fig. 7c).

\section{Discussion}

The morphologic analysis provides some interesting information that can be correlated with the values of capacitance. The samples with small amounts of PANI $\left(<1 \mu \mathrm{g} \mathrm{cm}{ }^{-2}\right)$ deposited, present high values of capacitance. This is indicative of a welldispersed polymer grown through the nanoscopic pores of the silica gel. The nanowires formed present a very large surface to expose to the solution. However, when the amount of PANI deposited is higher, the PANI wires start to overflow the silica pores, and the nuclei start to collapse as observed in the SEM image of Fig. 8d. The agglomerates are formed when the amount of PANI deposited is more than $1 \mu \mathrm{g} \mathrm{cm}^{-2}$. It produces a decrease of the PANI surface area, as was indicated by the trend of the mass capacitance of the electrodes (Fig. 6b).

The growth of PANI wires inside or outside the pores can be identified by the analysis of the chronoamperogram obtained during the electropolymerization. Two regimes were observed in these plots for ITO/silica electrodes, separated by an inflection point (critical time).

Before the critical time, a higher (compared with bare ITO), non-exponential rate of electrochemical polymerization is related to the growth of isolated PANI wires across the silica pores (see Scheme 1). Faster kinetics of polymerization may be related to a "confinement effect" of the aniline oligomers created upon the electrochemical pulse. The diffusion of oligomers to the bulk solution is hindered by the presence of the silica matrix. The high concentration of oligomers near the electrode surface induces a faster electropolymerization inside the silica matrix.

The resultant silica-PANI obtained in potentials steps shorter than the critical time presents a high electroactive area, since the

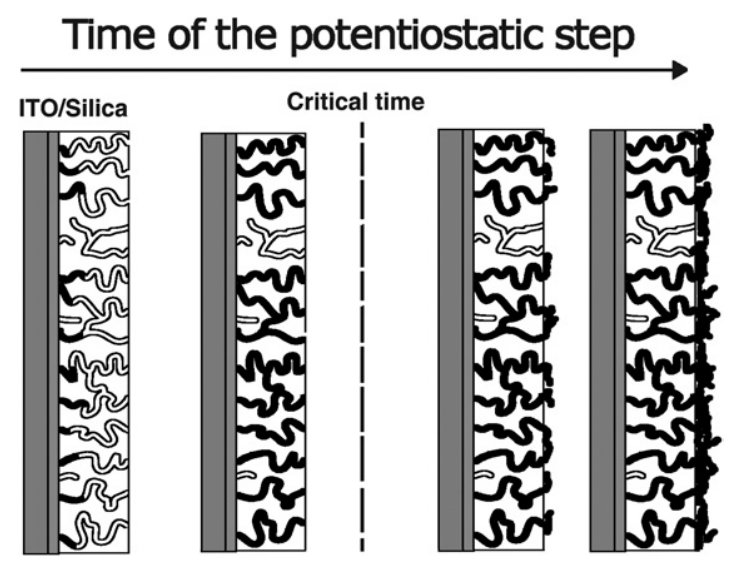

Scheme 1 
electrolyte is allowed to diffuse inside the silica pores, and the silica avoids electrical contact between conducting wires, maximizing, therefore, the area exposed to the solution.

However, the interpenetrating PANI-silica electrodes present higher amounts of defects (overoxidised sites) than the polymer grown on bare ITO (see defects ratio in Table 1). The oxidation of aniline monomers produces several oligomers, mainly dimers, in the vicinity of the ITO surface. As indicated, the "confinement effect" hinders the diffusion of these species to the bulk solution from the silica cavities. While the high concentration of oligomers has a positive effect on the kinetics of polymerization, the high oligomer-to-monomer ratio induces overoxidation of the former species followed by hydrolysis. ${ }^{21}$ It produces the appearance of defects in the polymer formed inside the silica pores.

After the critical time, the exponential growth of the current in ITO/silica electrodes indicates the overflow of the polymer that grows with similar kinetics to those on bare ITO surfaces, although the separation of the emerging nuclei produces a lower rate for composite electrodes than for conventional ITO ones.

\section{Conclusions}

Polyaniline-silica composites have been synthesized by electrochemical reactive insertion using potentiodynamic and potentiostatic methods. The shape of the chronoamperograms indicates two regimes for the electrochemical polymerization reaction. A fast growth of polymer happens within the silica pores at the initial stages due to confinement effects of oligomeric species formed upon oxidation. The growth of PANI overflowing the pores follows a similar behaviour to that on bare ITO electrodes.

The electrochemical capacitance of the silica-PANI composites is several times higher than the capacitance of ITO/PANI synthesized in the same conditions. These results are indicative of a higher surface area of the electroactive species exposed to the solution. The silica matrix avoids the contact and the electric collapse between vicinal conducting fibres of PANI increasing the conducting surface exposed to the electrolytic solution, compared with conventional ITO/PANI electrodes.

\section{Acknowledgements}

This work was financed by the following projects: MAT200760621 of the Spanish Ministerio de Ciencia e Innovación and GVPRE/2008/249 of the Generalitat Valenciana. F.M. acknowledges the financial support given by the "Ramón y Cajal" program.

\section{References}

1 E. Dovgolevsky, S. Kirmayer, E. Lakin, Y. Yang, C. J. Brinker and G. L. Frey, J. Mater. Chem., 2008, 18, 423.

2 S. W. Ho, T. K. Kwei, D. Vyprachticky and Y. Okamoto, Macromolecules, 2003, 36, 6894.

3 T. Q. Nguyen, J. J. Wu, V. Doan, B. J. Schwartz and S. H. Tolbert, Science, 2000, 288, 652

4 T. Q. Nguyen, J. Wu, S. H. Tolbert and B. J. Schwartz, Adv. Mater., $2001,13,609$

5 T. Aida and K. Tajima, Angew. Chem., Int. Ed., 2001, 40, 3803.

6 W. J. Doherty, N. R. Armstrong and S. S. Saavedra, Chem. Mater., $2005, \mathbf{1 7}, 3652$.

7 R. Gangopadhyay and A. De, Chem. Mater., 2000, 12, 608.

8 C. Brinker and G. W. Scherer, in Sol-Gel Science: The Physics and Chemistry of Sol-Gel Processing, Academic Press, London, 1st edn, 1990.

9 J. Widera, A. M. Kijak, D. V. Ca, G. E. Pacey, R. T. Taylor, H. Perfect and J. A. Cox, Electrochim. Acta, 2005, 50, 1703.

10 M. M. Collinson, C. G. Rausch and A. Voigt, Langmuir, 1997, 13, 7245.

11 R. Esquembre, M. L. Ferrer, M. C. Gutierrez, R. Mallavia and C. R. Mateo, J. Phys. Chem. B, 2007, 111, 3665 .

12 A. Walcarius, D. Mandler, J. A. Cox, M. Collinson and O. Lev, J. Mater. Chem., 2005, 15, 3663 .

13 M. J. Bleda-Martinez, E. Morallon and D. Cazorla-Amoros, Electrochim. Acta, 2007, 52, 4962.

14 S. L. G. Lissy, S. Pitchumani and K. Jayakumar, Mater. Chem. Phys, $2002,76,143$

15 J. H. Sung, S. J. Kim and K. H. Lee, J. Power Sources, 2004, 126, 258.

16 M. M. Verghese, K. Ramanathan, S. M. Ashraf, M. N. Kamalasanan and B. D. Malhotra, Chem. Mater., 1996, 8, 822.

17 X. L. Luo, A. J. Killard, A. Morrin and M. R. Smyth, Electrochim. Acta, 2007, 52, 1865

18 Y. Z. Xian, F. Liu, L. J. Feng, F. H. Wu, L. W. Wang and L. T. Jin, Electrochem. Commun., 2007, 9, 773.

19 S. Neves, C. P. Fonseca, R. A. Zoppi and S. I. C. de Torresi, J. Solid State Electrochem., 2001, 5, 412.

20 F. Montilla, E. Morallon, A. De Battisti and J. L. Vazquez, J. Phys. Chem. B, 2004, 108, 5036.

21 L. Duic, Z. Mandic and S. Kovac, Electrochim. Acta, 1995, 40, 1681.

22 S. L. Mu and J. Q. Kan, Electrochim. Acta, 1996, 41, 1593. 